\title{
ON THE SOLUTION OF LINEAR FUNCTIONAL EQUATIONS BY AVERAGING ITERATION
}

\author{
W. G. DOTSON, JR. ${ }^{1}$
}

Curtis Outlaw and C. W. Groetsch [4] have recently shown that if $T$ is an asymptotically convergent continuous linear self-mapping of a Banach space $E$, and if $f$ is in the range of $I-T$, and $0<\lambda<1$, and $V_{\lambda}=\lambda I+(1-\lambda)(T+f)$, then for each $x \in E$ the sequence $\left\{V_{\lambda}^{n} x\right\}$ converges to a solution $u$ of the equation $u-T u=f$. Since under these same hypotheses Browder and Petryshyn [1] showed that the sequence $\left\{(T+f)^{n} x\right\}$ also converges to a solution $u$ of $u-T u=f$, the Outlaw-Groetsch theorem essentially says that the averaged iteration $x_{n+1}=V_{\lambda} x_{n}=\lambda x_{n}+(1-\lambda)\left(T x_{n}+f\right)$ yields a conservative process. The purpose of the present paper is to establish some fairly general conditions under which $\left\{V_{\lambda}^{n} x\right\}$ will converge to a solution $u$ of $u-T u=f$ (even when $\left\{(T+f)^{n} x\right\}$ does not).

Suppose $E$ is a Banach space, $T: E \rightarrow E$ is a continuous linear operator, and $f \in E$. For $0<\lambda<1$ we define $S_{\lambda}=\lambda I+(1-\lambda) T, V_{\lambda}=\lambda I$ $+(1-\lambda)(T+f)$, and $A^{\lambda}=\left[a_{n j}\right]$ where $a_{11}=1, a_{1 j}=0$ for $j>1$, and for $n>1, a_{n j}=\left(\begin{array}{c}n-1 \\ j-1\end{array}\right) \lambda^{n-j}(1-\lambda)^{j-1}$ for $1 \leqq j \leqq n$, and $a_{n j}=0$ for $j>n$. It is easily seen that $A^{\lambda}$ is a lower-triangular, nonnegative, infinite matrix with each row-sum equal to one and each column-limit equal to zero. For $n>1$ we have the real polynomial $a_{n}^{\lambda}(t)$ defined by

$$
S_{\lambda}^{n-1}(t)=(\lambda+(1-\lambda) t)^{n-1}=\sum_{j=1}^{n} a_{n j} t^{j-1}=a_{n}^{\lambda}(t) .
$$

So, defining $A_{n}^{\lambda}=a_{n}^{\lambda}(T)$, we have $S_{\lambda}^{n-1}=A_{n}^{\lambda}$, since $T$ is a linear operator. Defining

$$
b_{n}^{\lambda}(t)=\left(1-\hat{a}_{n}^{\lambda}(t)\right) /(1-t), \text { and } B_{n}^{\lambda}=b_{n}^{\lambda}(T),
$$

we have, for $n \geqq 2, B_{n}^{\lambda}=(1-\lambda)\left[I+S_{\lambda}+S_{\lambda}^{2}+\cdots+S_{\lambda}^{n-2}\right]$, since $I-T=(1-\lambda)^{-1}\left(I-S_{\lambda}\right)$. Also, for $n \geqq 2$, we have

$$
\begin{aligned}
V_{\lambda}^{n-1} & =\left[S_{\lambda}+(1-\lambda) f\right]^{n-1} \\
& =S_{\lambda}^{n-1}+(1-\lambda)\left[I+S_{\lambda}+\cdots+S_{\lambda}^{n-2}\right](f) \\
& =A_{n}^{\lambda}+B_{n}^{\lambda} f,
\end{aligned}
$$

Received by the editors June 10, 1969.

1 This research was supported in part by the North Carolina Engineering Foundation. 
since $S_{\lambda}$ is linear. It now follows at once from Theorem 3 of [3] that if $T$ is asymptotically $A^{\lambda}$-convergent (i.e., $\left\{I, T, T^{2}, \cdots\right\}$ is an asymptotically convergent semigroup with $\left\{A_{n}^{\lambda}\right\}$ as a system of almost invariant integrals) then $\left\{B_{n}^{\lambda}\right\}$ forms a system of companion integrals for $\left\{A_{n}^{\lambda}\right\}$ with respect to $T$. Consequently, with the above observation that $V_{\lambda}^{n-1} x=A_{n}^{\lambda} x+B_{n}^{\lambda} f$ for all $x \in E$ and all $n \geqq 2$, Theorem 4 of [3] specializes to yield the following result.

Theorem 1. Suppose $T$ is an asymptotically $A^{\lambda}$-convergent continuous linear operator on the Banach space $E$, where $0<\lambda<1$, and suppose $f \in E$. Then, the following are true:

(a) If $f$ is in the range of $I-T$, then for any $x \in E$ the sequence $\left\{V_{\lambda}^{n} x\right\}$ converges to a solution $u$ of the equation $u-T u=f$.

(b) If, for some $x \in E,\left\{V_{\lambda}^{n} x\right\}$ has a subsequence $\left\{V_{\lambda}^{n_{i}} x\right\}$ which converges weakly to a point $y \in E$, then $y-T y=f$ and $\left\{V_{\lambda}^{n} x\right\}$ converges to $y$.

(c) If, for some $x \in E,\left\{V_{\lambda}^{n} x\right\}$ is contained in a weakly compact subset of $E$, then $\left\{V_{\lambda}^{n} x\right\}$ converges to a solution of the equation $u-T u=f$.

In order to apply Theorem 1 , one has to know only that the continuous linear operator $T$ is asymptotically $A^{\lambda}$-convergent for some $\lambda, 0<\lambda<1$. In this direction we have the following result.

THEOREM 2. Suppose $T$ is a continuous linear operator on a uniformly convex Banach space $E$, and suppose $\|T\| \leqq 1$. Then for any $\lambda, 0<\lambda<1$, $T$ is asymptotically $A^{\lambda}$-convergent.

Proof. By Theorem 5 of [3] it suffices to show that

(a) $T$ is asymptotically $A^{\lambda}$-bounded,

(b) $T$ is asymptotically $A^{\lambda}$-regular, and

(c) $\left\{A_{n}^{\lambda} x\right\}$ clusters weakly for each $x \in E$.

Since $\|T\| \leqq 1$ we have

$$
\left\|A_{n}^{\lambda}\right\| \leqq \sum_{j=1}^{n} a_{n j}\|T\|^{j-1} \leqq 1
$$

for all $n$, so that (a) is true. To get (b) we first observe that

$$
T A_{n}^{\lambda}-A_{n}^{\lambda}=S_{\lambda}^{n-1} T-S_{\lambda}^{n-1}=(1-\lambda)^{-1}\left[S_{\lambda}^{n}-S_{\lambda}^{n-1}\right],
$$

so that $T$ will be asymptotically $A^{\lambda}$-regular (as defined in [3]) if and only if $S_{\lambda}$ is an asymptotically regular operator in the sense of Browder and Petryshyn [2]. Since $T$ is nonexpansive $(\|T x-T y\|=\|T(x-y)\|$ $\leqq\|T\| \cdot\|x-y\| \leqq\|x-y\|)$ and has at least one fixed point (viz. 0 , since $T$ is linear), and since $E$ is a uniformly convex space, Theorem 5 of [2] gives us that for any $\lambda, 0<\lambda<1, S_{\lambda}$ is an asymptotically regular 
operator. Hence we get (b). Finally, since uniformly convex Banach spaces are reflexive, closed spheres in $E$ are weakly compact. Since for any $x \in E$ we have for all $n$

$$
\left\|A_{n}^{\lambda} x\right\| \leqq\left\|A_{n}^{\lambda}\right\| \cdot\|x\| \leqq\|x\|,
$$

it follows that for any $x \in E$ the sequence $\left\{A_{n}^{\lambda} x\right\}$ clusters weakly; and so we get (c). Q.E.D.

Corollary. Suppose $T$ is a continuous linear operator on a uniformly convex Banach space $E$, and suppose $\|T\| \leqq 1$. Then for any $\lambda$, $0<\lambda<1$, and for any $x \in E$ the sequence $\left\{S_{\lambda}^{n} x\right\}$ converges (strongly) to a fixed point of $T$.

Proof. By Theorem 2, $T$ is asymptotically $A^{\lambda}$-convergent. Since $T$ is linear, $(I-T)(0)=0$. Hence part (a) of Theorem 1 can be applied, with $f=0$. But for $f=0$ we have $V_{\lambda}=S_{\lambda}$, and, of course, solutions of $u-T u=0$ are fixed points of $T$. Q.E.D.

Remark 1. Setting $\lambda=1 / 2$ in the above corollary provides an affirmative answer to a conjecture of Outlaw and Groetsch $[4, \mathrm{p}$. 431].

REMARK 2. It is easily seen that there are continuous linear operators which satisfy the hypotheses of Theorem 2, but which are not asymptotically convergent operators, e.g., any rotation of a finitedimensional Euclidean space about the origin, or, in $l^{2}$, the shift operator $\left(x_{1}, x_{2}, \cdots\right) \rightarrow\left(0, x_{1}, x_{2}, \cdots\right)$.

\section{REFERENCES}

1. F. E. Browder and W. V. Petryshyn, The solution by iteration of linear functional equations in Banach spaces, Bull. Amer. Math. Soc. 72 (1966), 566-570. MR 32 \#8155a.

2. - The solution by iteration of nonlinear functional equations in Banach spaces, Bull. Amer. Math. Soc. 72 (1966), 571-575. MR 32 \#8155b.

3. W. G. Dotson, Jr., An application of ergodic theory to the solution of linear functional equations in Banach spaces, Bull. Amer. Math. Soc. 75 (1969), 347-352.

4. Curtis Outlaw and C. W. Groetsch, Averaging iteration in a Banach space, Bull. Amer. Math. Soc. 75 (1969), 430-432.

North Carolina State University, Raleigh, North Carolina 27607 